\title{
TRAILERS IN BETWEEN SHORT VIDEO FORMS FROM DIGITAL GAMES TO MOVIES AND BACK
}

\author{
Enrico GANDOLFI \\ Kent State University \\ Roberto SEMPREBENE \\ Luiss Guido Carli University
}

\section{ABSTRACT}

Promotional trailers represent a significant trend in most entertainment sectors. However, little efforts have been done in enlightening this expressive form when related to video games. This article aims to address such a lack by analyzing game/movie (inspired by digital games) trailers as a crossroad between digital entertainment and films. The main intent is to exploit a comparative lens to uncover leading traits of short videos according to the medium that that they are portraying. Therefore, three case studies - i.e. Assassin's Creed, Warcraft, and Overwatch - are presented and unpacked with a combination of design and semiotic tools (from Greimas' narrative program to game heuristics) and the concepts of symmetry, dissymmetry and asymmetry advanced by Roger Caillois. Results point to a cross-media coherence between different types of trailers, and future developments are suggested for exploiting them as an informing research object.

Keywords: Assassin's Creed, cross media, game trailers, movie trailer, Overwatch, Roger Caillois, tie-in, Warcraft.

Trailers could be considered a strategic crossroad between movies and video games. Both the media harness this expressive form for promotional and informative purposes, and audiences are increasingly involved in their re-formulation and interpretation, from honest trailers and glitch compilations to mash-ups and machinima. We could describe trailers as a "short audiovisual content that anticipates a media experience". Despite this definition may sound vague, it represents a starting point for dealing with such a heterogenous phenomenon. Indeed, in last years companies' efforts have thrived to the extent that several categories of trailers can be currently outlined. For instance, in digital entertainment this production spans cutscenes, gameplay sessions, sequences built on purpose, live-action 
with professional actors, and so on. Despite the rise of alternatives such live streaming and extended video coverages (e.g., on Twitch.tv and YouTube Gaming), trailers remain the reference way with which video games are framed with a non-interactive strategy. For instance, the trailer "The World of Pokémon GO has Expanded!" (available at https:// www.youtube.com/watch?v=R04u9E5INTI) had 3,102,080 views; while "Death Stranding" (Game Awards 2016) (available at https://www.youtube.com/watch?v=6goa1pl_az8\&t=131s) reached 1,954,224 viewers and "FOR HONOR ALL Heroes Class Gameplay Trailers" (available at https://gaming.youtube.com/watch?v=Aa4N_5CtUUc) 2,666,792 ones (by February 18 2017). Therefore, it is surprising that little academic efforts have been done in enlighten their traits and characteristics (for exceptions, see Carlson, 2009; Svlech, 2015). Such a lack is even more significant if we address the relation between movies and video games, in which trailers may work as a crossroad for staging a new comparative lens. It can be argued that the bridge between these two media was deeply explored (e.g., Bittanti, 2008; Bolter \& Grusin, 1999; Wolf, 1997), but short videos were always secondary variables within the overall equation.

This article aims to deal with this issue by conducting a comparative analysis of trailers of movies inspired by video games and the video games' trailers. Specifically, the framework outlined by Gandolfi and Semprebene (2015) will be adopted and refined to assess three case studies - i.e., Warcraft, Assassin's Creed, and Overwatch. Main references will span Game Studies, Media Studies and Semiotics. The wished contributions are twofold: providing more insights about this specific form of content, and suggesting an alternative path to compare the two media. The next pages are structured as follows: the first section introduces trailers as a specific medium; the second is about the analytic tools deployed to address them; the third depicts the research design, while the fourth present the three case studies: finally, the fifth regards conclusions and possible future developments.

\section{OVERVIEW}

While a video game is defined by interaction, video game trailers can adopt multiple forms: they may be CG (Computer Graphic) animated videos created ad hoc, gameplay trailers realized with the game's graphic engine, a mix of the two, or even a real live-action video -e.g. Destiny (available at https://www.youtube.com/watch?v=uxi18-ENKMM). Several factors are involved in choosing a video game trailer form, but it frequently happens that more than one are eventually realized, offering different materials to the marketers and satisfying divergent types of audiences. Moreover, the synthetic nature of the medium allows to have both virtual and real appearances in trailers: video games are a simulation of a possible reality and a live-action trailer might be an expression of it, while there would not be any point in creating a CG trailer for a live-action film. A trailer is indeed an invitation, a taste of something that makers want you to buy in its complete form. If the reference is to a realistic movie, a CG or animated short video would be questionable due to the weak relationship/ coherence between fictional aesthetics of the trailer and the realistic layout of the movie, which is a more closed (and less expandable) type of text. On the contrary, game trailers can span and focus on several aspects of a game, from main plot and gameplay to aesthetic beauty and game community, because of the multiple possible worlds (Juul, 2005) they refer to. It can be argued that the genre - i.e. a well-established group of features usually perceived as a whole - plays a crucial role in selecting the game peculiarities to promote. Nevertheless, these portrayable traits are higher in comparison with movie trailers due to 
the different nature of the source text. Video games are ergodic media characterized by an interactive layer that is missing in movies. Therefore, the formal complexity of the former is wider than the one of the latter, and trailers can target a more extended array of elements.

Despite such a complexity, game trailers have been poorly analyzed with broad and usually descriptive categorizations (Carlson, 2009; Svlech, 2015). Conversely, movie ones keep stimulating analyses and researches about their semiotic quality and commercial effectiveness (e.g. De Vries \& Lado, 2017; Johnston, 2009; Karray \& Debernitz, 2017; Liu et al., 2016). Among the most recent highlights, it is worth to notice that this form of advertising has proven to have a dramatic impact on the success of movie, and that interactive trailers are more effective than non-interactive ones. By following such suggestions, the present article aims to take a step forward in framing game trailers in combination with related theatrical ones.

\section{FRAMEWORK}

As mentioned above, the leading reference is the framework developed by Gandolfi and Semprebene (2015) about movies inspired by video games. According to its premises, 4 analytic dimensions should be addressed in investigating the interplay between these two media:

- Narrative: story and characters following the semiotic narrative program suggested by Greimas (1983). Accordingly, every narrative arc is composed by four sequences: the contract (the leading actor is informed about his/her mission); the competence ( $\mathrm{s} / \mathrm{he}$ is trained to succeed); the performance (s/he is finally put to test); and the sanction (the verdict). In addition, character's' core types (i.e., the so-called actants) span six different roles: the subject (the leading character), the object (the target of the action), the sender (who informs the hero about his/her mission), the villain (the opponent), the helper (who assists the hero in succeeding) and the receiver (who assesses the subject's performance in the end).

- [a] Aesthetic: figurative, plastic and technical features that inform the audio-visual content (Floch, 2012), from stylistic influences and graphics to colors and forms embedded in the aesthetic layout. It can be argued that the main orientation can be either realistic - i.e. reproducing reality - or iconic - depicting an abstract environment (Bateman \& Boon, 2006).

- [e] Encyclopaedian: the information/symbolic dimension that encompasses the audio-visual content and drives its understanding. Eco (1975) argues that any textual interpretation is affected by contiguous texts and experiences. It means that media content can benefit from such further connections and synergies (e.g. transmedia strategies) but also be undermined (e.g., a reference that is mandatory to understand to comprehend the text).

- [d] Dynamic: rules, patterns (Adams \& Dormans, 2012), and heuristics (Elias, Garfield, \& Gutschera, 2012) portrayed by and structuring the text. For instance, the logics and processes operating behind the protagonist's action, and how other actants react and perform. We can refer to this parameter as the agential rationale affecting the media content. 
Previously, these categories were deployed to assess the relation between digital games and their filmic transpositions following two different continuums - i.e., coherence - fidelity to the source game - and dependency - how the knowledge of the source game is necessary to understand the tie-in. In the present article, we will keep them referring to the shared brand that game and movie trailers have in common. However, we will harness the distinction between symmetry, dissymmetry and asymmetry sketched by Roger Caillois, who is one of the fathers of Game Studies, as further parameters.

The first can be described as a concordance between two elements. Symmetry is associable with mimicry and mirroring efforts. From a social perspective, we are referring to the status quo and the repetition of clichés. The second is the opposite - i.e., a force aimed to overturn standards and consuetudes. It is the vertigo, the revolution, the unexpected. For instance, a movie trailer that dramatically reformulates the game one. The third is the middle point between the two: after a dissymmetrical input, a return to a new symmetry is staged via an asymmetric process. It could be described as a restoration path.

According to Caillois, these phases characterize both human and natural domains. In his Diagonal Sciences holistic lens, universal principles regulate the whole reality without distinctions. This framework was already proven to be effective in game analysis (Gandolfi, 2016) although its use is still marginal in the field. Our conviction is that it can support crossmedia comparisons effectively because of its wide but critical orientation. For example, a medium can address a franchise differently from another and move forward the related interpretation. Otherwise, a symmetry might be developed between the two expressive forms and then a reiteration is staged.

Regarding our research object, this approach asks us to a) detect potential similarities and divergences between game and movie trailers toward the reference brand and $b$ ) observe variations in perceiving the video game franchise addressed in dealing with the source-game. Indeed, dissymmetry, symmetry, and asymmetry describe not just the relation characterizing two objects but also the outcome of their interaction. We will focus on the latter definition in these pages. Caillois (1984: 27) notices that symmetric behaviors and especially mimicry bring to «an incantation fixed at its culminating point» that weaken and remove peculiarities, subjectivity, and difference. Therefore, «life takes a step backwards» (30) and a devouring inertia thrives. On the contrary, dissymmetry fosters creativity, innovation, and progress. Caillois (1976: 247) claims that it is at the core of the Darwin's law of evolution: an unexpected anomaly affects structures that were considered untouchable, and a new turn can be triggered and flourish. Finally, such a novel input become increasingly structured i.e. an asymmetry - eliciting symmetries and then a new order (waiting for the next change).

\section{APPLYING THE FRAMEWORK}

In dealing with our research object, the challenge is to understand how the movie trailer follows or reformulates the game one. Such a specific focus, which considers the extended texts (i.e. movies and game) general references, aims to give these shorts videos a proper attention, which is still missing. By comparing and analyzing some pertinent examples, the objective is to better understand their peculiarities and advocate for more studies in this direction. However, we will obviously consider the context of production and consumption to outline a satisfying overview, especially considering the evolution of the targeted franchise. 
We have selected two ideal cases - i.e. Assassin's Creed and Warcraft - and a further hybrid one - i.e. Overwatch - for stressing our assumptions. Assassin's Creed is a popular action-game franchise owned by Ubisoft. The first chapter was released in 2007 and the last installment in 2016 for a total of 9 main episodes and over 100 million copies sold. The tiein was out in 2016 after 5 years of planning and production. Directed by Justin Kurzel and starring Michael Fassbender, Marion Cotillard and Jeremy Irons, it received negative-mixed reviews and raised $\$ 241.4$ million against a budget of $\$ 125$ million.

It can be argued that Warcraft is one of the most acclaimed brands in digital entertainment. The first three strategic games are considered masterpieces, and World of Warcraft is the leading MMORPG (Massive Multiplayer Online Role-Playing Game) despite it was published in 2004, with more than 100 million accounts created so far. In addition, the digital carding game Hearthstone: Heroes of Warcraft was able to involve over 50 million players in two years. The Activision-Blizzard's franchise has informed several crossmedia expansions, among which the movie Warcraft in 2016 with Travis Fimmel, Paula Patton and Ben Foster. It costed $\$ 160$ million and grossed $\$ 433.7$ million, while reviews were generally mixed.

In the end, Overwatch is an online cooperative FPS (first person shooter) published by Activision-Blizzard in 2016. It was well received by critics and players with 7 million users reached in the opening week. Moreover, several trailers have been released to introduce the characters available in the game. These short videos are strongly influenced by Pixar's productions, from UP (2009) to Wreck-it Ralph (2012), and represent an exception in the sector because of their high quality. These three cases were selected due to their relevance in terms of visibility and centrality. Assassin's Creed and Warcraft are the most recent major movies inspired by video games, and Overwatch is a popular hybrid example that can help in stressing our analysis. We picked the main official trailers as leading reference to analyze. For Overwatch, we decided to focus on the first gameplay trailer and one of short videos mentioned above.

\section{CASE STUDIES}

\subsection{Assassin's Creed}

4.1.1. Assassin's Creed reveal trailer (available at https://www.youtube.com/ watch?v=RjQ6ZtyXoA0)

We decided to analyze the trailer of the first chapter of Assassin's Creed saga, released in 2007. Being a debut, it had both to explain the context and introduce the leading actors. The trailer lasts 2 minutes and is composed as a single coherent scene in CG. We see a man in disguise- i.e. Altair, the main character - watching an execution from the top of a bell tower in a medieval city. Suddenly, he disappears and we find him getting close to the execution platform and killing the hangmen and their captain with a hidden blade. He then run away, starting a parkour-style sequence that leads him to the entrance of a monastery. He seems to be surrounded by the surviving guards, but he manages to escape by mingling with a group of monks getting out from the abbey's doors in that precise moment. The trailer has a relevant cinematographic aura - the sequences are edited not just like a game session, but with attention to the rhythm, putting in the video all the aspects relevant to 
the comprehension of the setting - but it manages to express almost all the skills the player will use during the game: stealth movements, parkour, fighting and killing, and camouflage. Moreover, it portrays the contest, presenting a medieval Jerusalem and the enemies we will fight (among them is clearly visible a templar, anticipating an important aspect of narration). It is worth to notice that the trailer does not explain anything about the story behind the saga (e.g., the crucial role of Animus, protagonist's motivations), and no reference to the present as a part of the game is included. The trailer just creates the atmosphere and lets the viewer/player understand that the game is an action/adventure based on combat, stealth and acrobatics. According to our framework, the trailer is coherent to the game in all the 4 dimensions we considered: the narration is an example of what an Assassin actually does; the aesthetic is borrowed from the game itself; the information/symbolic dimension introduces the game references; and, although being a non-interactive video the trailer cannot be fully coherent with the kind of fruition the game offers, it actually enables the user to understand it, creating a strong link with the game on the Dynamics aspect. Moreover, the trailer sets the stage for a dissymmetry occurring when the player will access the final game. By hiding the contemporary part, it contributes to trigger a surprising effect with the final product, whose first minutes are set in the present.

\subsubsection{Assassin's Creed movie (available at https://www.youtube.com/watch?v=ecZnE6zu6_E)}

This theatrical trailer starts with a short but very meaningful scene of the main character (played by Michael Fassbender) who wakes up in a bed and widely opens his eyes. The detail of the pupil lead us to a scene where we can clearly recognize an Assassin as they appear in the game. From the very first scene present and past events are linked, something the game trailer did not specify, while in the film trailer is redundantly underlined. The film, and consequently the trailer, focus its attention on the present time, while the core of the game is set almost totally in the past. It is a difference that has an impact on all the four dimensions, because it represents a change of focus from the action in the video game to the background story in the film. Once the film project the viewer in the past, the aesthetics of the game, as well as the narrative, mechanics and encyclopedian are reproduced with great coherence (settings, outfits, movements, weapons) even if the historical setting is different from any of the video game episodes. We can notice a sort of asymmetrical movement in this trailer, which glaringly depicts the here-and-now setting. After 10 years from the first release, it is not a secret anymore; however, trailers of $A C$ episodes still focus on the historical setting. The movie one is more symmetrical in comparison with the game under this aspect (encyclopedian) and suggests new potential elements that are portrayed on the big screen. Regardless, the agential (stealth approach) and aesthetic (elegance of parkour skills) dimensions are kept coherent and familiar in both the trailers. It can be argued that such features were among the dyssmetrical strengths of the first Assassin's Creed, and now they are widespread in the sector, from Watchdogs to Infamous: Second Son. They remain the saga's identification mark aside from the medium chosen. 


\subsection{Warcraft}

4.2.1. World of Warcraft trailer (available at https://youtu.be/olc5-wQg6Og?list=PL9gZzeM4 mz7YgA3k34Xyv_hheCLXZyUls)

We select the first official CG trailer of World of Warcraft (WOW). Among the several Warcraft games, we chose this one because of its success and diffusion, which make it the reference installment of the brand. It lasts 3 minutes, starting with an overview of the game map and then presenting the different races and classes in their own environments. Finally, it combines them in 1 vs 1 fights in which their abilities are shown (e.g., dwarves' use of riffles, agility of elves, magic powers of magicians, and so on). The passage is from description to action, and the relation with the final game is characterized by an overall coherence and independence (the reference is to a familiar fantasy). Despite the main objective of the trailer is to get viewers familiar with the world narrative of the final product, it also shows some patterns of action that will take place in the interactive experience. There is not a specific protagonist but many, and this choice mirrors the multiplayer orientation of the game. It is also worth to notice that teamwork and cooperative gaming are not mentioned despite their centrality in WOW. There is an emphasis on competitive combat that is just one aspect of its gameplay. To summarize, the trailer seems to stress the clash of races behind the main plot. Probably it could depend on the fact the trailer was released when the cooperative aspects of the game were still not the main focus, having it to expand its audience. In a second phase the guilds element and the cooperation would have become more and more relevant. Aside from this focus, all the four dimensions are respected and coherent with the final experience.

\subsubsection{Warcraft: the Beginning (available athttps://www.youtube.com/watch?v=RhFMIRuHAL4)}

The trailer is 2:13 minutes long and depicts the plot premises. First, it introduces to the two leading protagonists of the movie - i.e. the orc Durotan and the human Lothar - and their respective worlds. Then, it develops the narrative arc, from the initial diffidence between them to the final alliance against a common enemy. Aesthetics (e.g., outfits, colors), plot (characters, narrative programs), dynamics (e.g., the aggressive style of Orcs) and encyclopedia (e.g., names, setting) are loyal to the brand and especially the first episode (Warcraft: Orcs \& Humans, published in 1994), whose events are at the core of the movie. This choice limits the variety of references at stake, but improves the nostalgia appeal with some frames (e.g., orc villages, armies) that are glaring tributes to the original RTS (Real Time Strategy). It means that several details might be missed or misunderstood if the viewer is not familiar with Warcraft, especially from an encyclopedian perspective. Following this influence, the trailer depicts the leading actants (protagonists, helpers, villain) accurately (e.g., motivations, values) and portrays a war rather that single fights. The consequent epic halo echoes that raids and extended battles that characterize WOW and the related teamwork (also fostered in WOW's expansions trailers). These two trailers are coherent to the brand and its world narrative. The game video represents an ideal introduction to the final product, while the theatrical one retrieves the beginning of the main story. Looking at the latter, it seems that the cooperative dynamic is getting central in portraying the franchise. The former was indeed missing such a mechanic, and it is interesting that the tie-in stresses such an attitude. It can be argued that the game trailer was influenced by the competitive dimension of the 
strategic chapters, while year after year the social-collaborative features of the MMORPG flourished influencing the overall meaning of Warcraft. Therefore, we can argue that we are observing the culmination of an asymmetry (from competition to teamwork, from enemies to allies), which becomes a new symmetry. Working with peers is the necessary competence to succeed toward the object of value - i.e. mastering the game or defeating the enemy in the movie.

\subsection{Overwatch}

\subsubsection{Gameplay trailer (available at https://www.youtube.com/watch?v=dushZybUYnM)}

This gameplay trailer is made by in-game footage and in 5:56 minutes explains the ability of different heroes (i.e. the different characters that a player can pick) during a 6vs6 match. For each, a brief introduction is given with name and outfit in stop-motion, and then its abilities are highlighted within the game context (e.g. Symmetra who sets traps for her enemies). The passage between protagonists is fluid and ruled by a paper-scissors-stone logic: the next hero kills the previous one by exploits his/her powers. The emerging effect is of a fast but variegated gameplay, in which any role can make a difference and a best strategy is missing. Being a gameplay trailer, the coherence with the game is taken for granted as well as the dependency. However, it underlines how difference and flexibility are the key of success. The game appears as a multi-layer experience, in which every actor can be the protagonist if the related "competence" is handled.

\subsubsection{Animated short "Dragons" (available athttps://www.youtube.com/watch? $v=0 J 09 x d x z \mid J Q$ )}

The animated short video "dragons" portrays the comparison between two scions of a Japanese clan - i.e. the samurai Hanzo and the cyborg-ninja Genji- who are both selectable heroes in the game. In its 8 minutes we watch the return of Hanzo to the clan headquarter and his battle with Genji, a sort of brother and main mimesis. The aesthetic is glaringly influenced by Pixar movies such as UP and Wreck it Ralph, and the mood of the trailer is both spectacular and hilarious mirroring the gameplay itself, without gore but characterized by a cheerful atmosphere. The skills deployed by the leading actants are the same ones available to their interactive counterparts in the game, and the setting/encyclopedia is general enough to be understood by newcomers (e.g., the Kill Bill movies) - a wise choice considering that this trailer was released almost with the game. An alternation between cartoon-style sequences and $2 \mathrm{D}$ rice-paper drawings is staged strengthening the coherence with Overwatch's layout and the oriental origin of the protagonists, who fight against each other without prevailing. Consequently, a further coherence with the multi-angle competence of the game is staged. Hanzo and Genji are the two sides of the same coin like any other characters in game - a sort of ludic yin and yang. Such a redundancy (also present in the other trailer analyzed) supports the asymmetrical passage carried out by this game, which has legitimized the use of multiple roles in online gaming as never before (Dota and Leagues of Legends set the stage for such a mechanic, but Overwatch made it a new standard). It can be observed that Activision- Blizzard has often followed this approach - i.e. concretizing a rising asymmetry into a widespread symmetry: think about its World of Warcraft, Starcraft and Diablo, which wrote in stones the leading features of their own genres. 


\section{CONCLUSIONS}

Video games opened to a totally new interpretation for the idea of "trailer", a short promotional video that can adopt multiple solutions, from the in-game graphics to the liveaction. Compared to cinema, video games offer a previously unknown freedom to explore different languages and forms, even the length has potentially a wider choice, being the video game trailers mainly viewed on internet. These possibilities are strictly connected to the varied and interactive nature of the medium, which allows the trailer to refer to different aspects of the narrative worlds they talk about. When this narrative universe is declined in film, the trailer has to follow it, getting back to the structure defined by a fruition that is bound to commercial spaces (generally limited to $30^{\prime \prime}, 60^{\prime \prime}, 90^{\prime \prime}$ and $120^{\prime \prime}$ ) and to a story which is unique and set - it is possible to shoot a dedicated video, but generally cinema trailers have to be realized with film shooting, not giving the chance to explore something different and in different languages. This passage strengthens the idea of trailers as meaningful forms that are able to bring information despite their brevity (Pezzini, 2002; Peverini, 2004).

The desired outcome of this article is to have provided a set of tools for unpacking such a specific type of medium in comparison with other related trailers and targeted franchises. By assessing movie and game short videos, the aim is to sketch a multi-angle lens on this phenomenon. Trailers condense and summarize brand identity and have a significant range of spreadness that may go beyond the products depicted. Our conviction is that the framework applied works also in addressing this research object, while the cycle symmetryasymmetry-dissymmetry helps in better understanding the consequent impact in relation with the game brand. Such a combination seems effective even in deepening Overwatch, which was studied with different criteria of comparison (i.e., the game itself). Among the results, it can be argued that in all the examples considered movie and game trailers are connected traces - milestones of the franchise's evolution. Sometimes they work together (Overwatch), sometimes they move ahead the saga's interpretation (Assassin's Creed), sometimes they fix each other (Warcraft) despite their obvious differences and the divergent source promoted. Nevertheless, a sort of long-term coherence seems to occur, reiterating the idea of a transmedia breath that trailers can easily demonstrate with their summarizing focus.

Regardless, this study remains an exploratory attempt to address a still overlooked theme. More case studies are necessary to stress, validate, and problematize our proposal, and the reference is to a constantly evolving sector, which requires an ongoing analytic attention. Regardless, we hope to have provided enough stimuli to proceed in enlightening game trailers and their constant evolution in relation with contiguous media. Future developments might address different types of short video forms (e.g., honest trailers, glitch compilations) and users' reactions and endorsements (e.g., online discourses and comments on Youtube and other social media).

\section{REFERENCES}

ADAMS, W. E. \& DORMANS, J., Game Mechanics: Advanced Game Design, San Francisco, New Riders, 2012.

BATEMAN, C. \& BOON, R., 21st Century Game Design, Hingham, Charles River Media, 2006.

BITTANTI, M. (ed.), Schermi interattivi. Il cinema nei videogiochi, Rome, Meltemi, 2008. 
BOLTER, J. D. \& GRUSIN, D., Remediation: Understanding New Media, Cambridge, The MIT Press, 1999.

CAILLOS, R., «Mimicry and legendary psychasthenia», October, 1 (1935/1984), pp. 17-32.

CAILLOIS, R., Cohèrences aventureuses, Paris, Gallimard, 1976.

CARLSON, R., «Too Human versus the enthusiast press: Video game journalists as mediators of commodity value», Transformative Works and Cultures, 2 (2009), available at http:// journal.transformativeworks.org/index.php/twc/article/view/98/93

DE VRIES, E. L. E. \& LADO, N., «Abstract: Seduced at the Movies: Interactive Cinema Advertising Enhances Advertising Effectiveness by Lowering Construal Level for HighInvolved Consumers», in Stieler M. (eds.), Creating Marketing Magic and Innovative Future Marketing Trends. Developments in Marketing Science: Proceedings of the Academy of Marketing Science, Berlin, Springer, 2017.

ECO, U., Trattato di semiotica generale. Milan, Bompiani, 1975.

ELIAS, S. G., GARFIELD, R. \& GUTSCHERA, K. R., Characteristics of Games, Cambridge, The MIT Press, 2012.

FLOCH, J. M., Identità visive. Costruire l'identità a partire dai segni (third edition), Milan, Franco Angeli, 2012.

GANDOLFI, E. \& SEMPREBENE, R., "Play the game in the opening scene A multidisciplinary lens for understanding (video)ludic movies, including Super Mario Bros., Resident Evil and Scott Pilgrim vs. the World», G/A/M/E, 4 (2015), available at http://www.gamejournal. it/gandolfi_play/

GANDOLFI, E., «Beyond Diagonal Sciences: Applying Roger Caillois's Concepts of Symmetry and Dissymmetry to Journey», Games and Culture, (2016), Epub ahead of print September 14, 2016, doi: 10.1177/1555412016667342.

GREIMAS, A. J., Structural Semantics: an Attempt at a Method. Lincoln, University of Nebraska Press, 1983.

JOHNSTON, K. M., Coming Soon: Film Trailers and the Selling of Hollywood Technology, Jefferson, Mcfarland \& Co Inc Pub, 2009.

JUUL, J., Half real, Cambridge, The MIT Press, 2005.

KARRAY, S. \& DEBERNITZ, L., "The effectiveness of movie trailer advertising», International Journal of Advertising, 36 (2017), pp. 368-392.

LIU, S. et al., «What Makes a Good Movie Trailer?: Interpretation from Simultaneous EEG and Eyetracker Recording», in Hanjali A. \& Snoek C. (eds.), Proceedings of the 2016 ACM on Multimedia Conference, New York, ACM, 2016.

PEVERINI, P., II videoclip. Strategie e figure di una forma breve, Milan, Meltemi, 2004.

PEZZINI, I., Trailer, spot, clip, siti, banner. Le forme brevi della comunicazione audiovisiva, Milan, Meltemi, 2002.

ŠVELCH, J., «Towards a typology of video game trailers: Between the ludic and the cinematic», G/A/M/E, 4 (2015), available at http://www.gamejournal.it/svelch_trailers/

WOLF, M. J. P., «Inventing Space: Toward a Taxonomy of On- and Off- Screen Space in Video Games», Film Quarterly, 51 (1997), pp. 11-23. 